\title{
A fundamental limitation on growth rates in the traveling heater method
}

\author{
Jeffrey H. Peterson, Andrew Yeckel, Jeffrey J. Derby* \\ Department of Chemical Engineering and Materials Science, University of Minnesota, 151 Amundson Hall, \\ 421 Washington Avenue SE, Minneapolis, MN 55455-0132, USA
}

\section{Abstract}

A comprehensive mathematical model of the traveling heater method is applied to understand interactions of flow, segregation, and stability for the crystal growth by the traveling heater method. We demonstrate that the formation of a secondary, counter-rotating vortex near the growth interface limits the transport of segregated solvent, leading to large concentration gradients and supercooling of the liquid near the interface. The secondary vortex arises from the same mechanism responsible for the formation of lee waves in atmospheric flows, and its spatial form scales with the Brunt-Väisälä frequency. Significantly, this supercooled layer of liquid arises from the lateral transport of solute, which is predominantly affected by the secondary flow vortex, rather than the axial diffusion of solute, as assumed in the classical derivation of constitutional supercooling by Mullins and Sekerka. Thus, supercooling in a model cadmium zinc telluride system occurs at growth rates of order $1 \mathrm{~mm} /$ day, nearly an order of magnitude smaller than expected from classical arguments. Paradoxically, the traditional strategy to alleviate constitutional supercooling during crystal growth, namely increasing the temperature gradient at the interface, is expected to strengthen the lee-wave vortex and accentuate the onset of instability. Other approaches will be needed to overcome growth limits in the traveling heater method.

Keywords: A1. Computer simulation, A1. Convection, A1. Mass transfer, Heat transfer, A2. Traveling heater method growth, A2. Traveling solvent zone growth, B2. Semiconducting II-VI materials

${ }^{*}$ Corresponding author. E-mail address: derby@umn.edu (J.J. Derby). 


\section{Introduction}

The traveling heater method (THM) is an important technique for producing compound semiconductor crystals, particularly detector-grade cadmium zinc telluride (CZT) [1-3], and it promises a number of advantages over classical Bridgman methods [4, 5]. Unlike the Bridgman method, the THM uses a melt zone that is enriched in one component of the compound, which acts as a solvent. As the ampoule travels past a heater, the liquid zone dissolves a solid feed while simultaneously growing material of a similar composition at the opposite end of the hot zone. Since the crystal grows from a liquid flux, the THM allows for operation at much lower temperatures than the Bridgman process, and its path through the phase diagram results in comparatively better stoichiometry. This may be especially important for the growth of CdTe and alloys, where less excess tellurium within the grown crystal [6] may favorably limit the formation of tellurium precipitates.

Despite the strengths of the THM, it is currently hampered by growth rates that are approximately an order of magnitude slower than those routinely employed in Bridgman methods $[1-3,7,8]$. At present, this growth rate limitation is not well understood; however, constitutional supercooling has been put forth as a possible explanation [9] .

In the classical scenario of constitutional supercooling during directional solidification [10], species segregation leads to either a depletion or accumulation region in front of the advanc-

ing interface. A concentration gradient is then formed that changes the local melting point within the liquid. As the concentration gradient increases with higher growth rates, a critical value will be reached where the compositionally-dependent melting point is driven higher than the local temperature in the melt in front of the interface, thereby leading to supercooled liquid and constitutional supercooling. The growth rate limit for stable growth can be estimated as,

$$
V_{\mathrm{g}, \max }<\frac{-\mathcal{D}}{m C(1-K)} \frac{\mathrm{d} T}{\mathrm{~d} z},
$$

where $\mathcal{D}$ is the species diffusivity, $m$ is the slope of the liquidus line, $C$ is the liquid species concentration at the interface, $K$ is the partition coefficient, and $\mathrm{d} T / \mathrm{d} z$ is the thermal gradient in the liquid normal to the interface. Using typical values for the THM growth of CZT-assuming the segregated species to be tellurium and the temperature gradient 
at the growth interface to be $70 \mathrm{~K} / \mathrm{cm}$ - this maximum growth rate is calculated to be approximately $10 \mathrm{~mm} /$ day, which is an order of magnitude higher than the maximum growth rates that are actually observed for THM growth of CZT.

Here, we apply a comprehensive computational model [11] to address the question of why such stringent growth rate limits arise for the traveling heater method. Past models for this process have suggested that morphological instability could readily arise in THM $[12,13]$. However, the seminal work of Lan and Yang [12] was limited in its ability to consider terrestrial growth by the computational resources needed to resolve flow structures in the zone. More recent work by Stelian and Duffar [13] has also suggested morphological instability limitations but did not clarify their underlying cause. In the following discussion, we put forth a new physical mechanism that may be responsible for THM growth limitations. Namely, we demonstrate that a classical fluid mechanical instability in the liquid zone can result in constitutional supercooling at growth rates far lower than predicted by classical theory.

\section{Model}

For the sake of brevity, we will briefly outline our model for the traveling heater method; a more detailed discussion is presented in [11]. Continuum transport and interfacial phenomena within the ampoule are represented in a local model. This model represents heat transfer through all domains along with fluid flow and mass transport in the liquid zone. The positions of the dissolution and growth interfaces are computed in a self-consistent manner with the underlying field equations, so that mass and heat fluxes are balanced, satisfying local segregation and equilibrium conditions along the interfaces per requirements of the CdTe phase diagram. We consider this cylindrical system to give rise to two-dimensional, axisymmetric behavior. For a well-defined steady state, we also specify the total amount of excess tellurium contained in the liquid zone. The local model is schematically shown in the bottom portion of Figure 1.

To provide appropriate thermal boundary conditions to the local model, a global model for conductive and radiative heat transfer in the furnace is computed using the CrysMAS code [14], developed by the Fraunhofer IISB. The top portion of Figure 1 shows the thermal field 
as well as the dimensions and materials for the furnace, along with an appropriate thermal load substituted for the ampoule. The temperature and heat flux are then measured along the ampoule surface and are approximated by an effective radiation temperature that is employed in a flux boundary condition to the local model.

To solve for the resulting interface geometries, as well as thermal, flow, and composition fields, we make use of the Cats2D Galerkin finite element code [15] that has been used previously for a number of different crystal growth systems [16-22].

\section{Results}

We consider an ampoule with a diameter of $4.8 \mathrm{~cm}$, made from 2-mm thick quartz. Material properties used in the simulations are presented in [11]. The traveling heater produces a thermal gradient of approximately $70 \mathrm{~K} / \mathrm{cm}$ at the growth interface. The equations for mass, momentum, and energy transport were then solved over a computational domain with 192,480 unknowns. Mesh resolution studes were performed to assure accurate solution of the underlying model equations.

We present an extensive discussion of the mechanistic underpinnings of the THM in [11]. Below, we highlight computations that demonstrate the formation flow structures in the liquid zone, specifically lee waves, and show their relevance to constitutional supercooling effects that limit stable growth.

\subsection{Flow, transport, and shape of liquid zone}

We consider both solutal and thermal effects on liquid density; however, we find that thermal effects dominate. Thus, the temperature field, in the presence of gravity, predominantly drives convective flows in the liquid zone. Specifically, the localized heating of the zone results in radial temperature gradients that drive strong flows, with liquid moving upward along the outer domain, adjacent to the inner ampoule wall, and moving downward along the system centerline. This primary, central flow cell is shown via streamlines in Figure 2.

The strong primary flow significantly affects both heat and mass transport though the zone, and the effects of convective transport are clearly seen in the temperature and concentration fields in Figure 2. In particular, isotherms in the liquid are strongly deflected, 
taking on the general shape of flow streamlines. Heat carried by the flow along the centerline results in downward deflection of the growth interface. The excess tellurium in the

liquid zone is nearly perfectly mixed within the bulk of the zone; however, thin boundary layers persist along the dissolution and growth boundaries, as indicated by the closely spaced iso-concentration lines.

There is a feature of extraordinary interest in this system, notably a counter-rotating, secondary vortex nested along the growth interface. This feature affects the tellurium concentration field, producing a noticeable deflection of tellurium iso-concentration lines. The significance of this feature on the growth of the crystal is elaborated below. For completeness, we also note that a small vortex arises in the upper, outer corner of the liquid zone. While this vortex affects tellurium transport from the dissolution interface, it does not strongly influence growth.

\subsection{Segregation and constitutional supercooling}

Excess tellurium accumulates in front of the growth interface as it is rejected from the growing solid. If there were only the primary convection cell, liquid would uniformly flow outward across the interface, and the excess tellurium would simply be swept along the interface, toward the outer periphery of the growing crystal, and into the bulk. However, the secondary, counter-rotating vortex at the growth interface reverses the flow over a portion of the interface, creating a resistance to mass transport and promoting the local accumulation of tellurium.

This situation is depicted in Figure 3, where there is a substantial accumulation of tellurium near the secondary vortex. Indeed, this accumulation leads to large concentration gradients within the solutal boundary layer. When these gradients are large enough, the composition-dependent melting point increases faster than the temperature field beyond the growth interface. This results in an extended region of supercooled liquid immediately in front of the growth interface, leading to constitutional supercooling and an unstable growth situation. 


\subsection{Lee waves}

Why does this counter-rotating vortex form at the growth interface in this system? The answer lies in the particular nature of the thermal field. Radial thermal gradients drive a strong flow in the liquid zone, due to the lever-arm forces arising from gravity acting on radial density differences. However, there are also significant axial thermal gradients that affect the density of the liquid. As the growth interface is approached, the temperature decreases, resulting in denser liquid. The situation of denser underlying less-dense fluid is stable in a gravitational field and is referred to as stable stratification.

A classical flow instability associated with stable fluid stratification is the formation of lee waves [23]. Lee waves have traditionally been understood in the context of atmospheric phenomena [24] but can be present in any stably-stratified flow. A lee wave is formed when a stably stratified flow is subjected to a perturbation in the direction of the density gradient. This perturbation then propagates in the flow, producing a characteristic wave with a welldefined frequency $N$, known as the Brunt-Väisälä frequency. The term lee wave refers to a common situation observed in nature, where such waves form downwind, i.e., in the lee, of a mountain range and are manifest by ordered cloud patterns, which often break up into interesting forms, such as lenticular clouds.

Returning to the THM system, when the primary flow cell drives fluid downward along the centerline of the liquid zone, it feels a resistance from the stable vertical stratification of density. As the liquid turns to flow outward along the growth interface, the kinematics associated with this vertical density gradient produce a lee wave and an accompanying, counter-rotating vortex. In this situation, the turning of the flow acts as a perturbation, and, due to the outer turning of the flow by the ampoule wall, there is not enough lateral distance for more than a single lee wave to form.

Figure 4 presents a series of calculations meant to validate our assertion that the leewave mechanism is responsible for the counter-rotating vortex at the growth interface in THM. In these computations, the thermal gradient near the interface was increased by modifying the form of the heater profile while keeping all other features unchanged. Using the Boussinesq approximation to represent changes in liquid density with temperature, the 
Brunt-Väisälä frequency is given by,

$$
N=\sqrt{g \beta \frac{\partial T}{\partial z}},
$$

where $g$ is the gravitational constant, $\beta$ is the thermal expansion coefficient of the liquid, and $\partial T / \partial z$ is the vertical temperature gradient at the growth interface. According to this relation, as the thermal gradient increases, the frequency of the oscillation also increases and the wavelength associated with the flow structure, which scales inversely with frequency, must then decrease. Intuitively, this can be explained by thinking about the system as a harmonic oscillator: a stronger thermal gradient represents a stiffer spring that will oscillate at a higher frequency. This outcome is nicely demonstrated by the change in position of the counter-rotating vortex for the different cases shown in Figure 4. With increasing vertical temperature gradient, the vortex position shifts toward the centerline, with a shorter wavelength $\lambda$, and increases in strength, with a larger streamfunction maxima $\psi_{\max }$. This behavior is examined in more detail, with a quantitative demonstration of how the lee-wave position scales consistently with equation (2), as $\lambda \propto(\partial T / \partial z)^{-1 / 2}$, in a future publication [25]. Note that the lee-wave structure arises solely from thermal effects and is not directly influenced by the growth rate.

\section{Conclusions}

Via insight obtained from our computational model, we put forth a plausible explanation for the physical mechanisms that limit the growth rates attainable in the traveling heater method. We posit that supercooling is promoted in the liquid near the growth interface by the formation of a counter-rotating vortex, which arises from the same mechanism responsible for the formation of lee waves characterized by a Brunt-Väisälä frequency. This vortex limits the transport of segregated solvent, tellurium in this case, leading to large concentration gradients and supercooling. The liquid supercooling will subsequently lead to morphological instability and growth rate limitation.

Significantly, this supercooled layer of liquid arises from lateral transport of solute, which is predominantly affected by the secondary flow vortex, rather than the axial diffusion of solute, as assumed in the classical derivation of constitutional supercooling [10]. Thus, 
supercooling in this system occurs at growth rates far lower than predicted by equation (1). Here, the instability is manifested for growth rates of order $1 \mathrm{~mm} /$ day, nearly an order of magnitude smaller than expected from classical arguments.

Paradoxically, the traditional strategy to alleviate constitutional supercooling during crystal growth, namely increasing the temperature gradient at the interface, is expected to strengthen the lee-wave vortex and accentuate the onset of instability. Other approaches will be needed to overcome growth limits in the traveling heater method. We anticipate that insight provided by comprehensive models, such as that employed here, will be invaluable toward evaluating new growth strategies.

\section{Acknowledgments}

This work is supported by the U.S. National Science Foundation NSF DMR-10007885, the content of which does not necessarily reflect the position or policy of the United States Government, and no official endorsement should be inferred.

\section{References}

\section{References}

[1] M. Funaki, T. Ozaki, K. Satoh, , R. Ohno, Growth and characterization of CdTe single crystals for radiation detectors, Nuclear Instruments and Methods in Physics Research Section A: Accelerators, Spectrometers, Detectors and Associated Equipment 436 (1999) $120-126$.

[2] H. Chen, S. Awadalla, J. Mackenzie, R. Redden, G. Bindley, A. Bolotnikov, G. Camarda, G. Carini, R. James, Characterization of traveling heater method (THM) grown Cd0.9Zn0.1Te crystals, Nuclear Science, IEEE Transactions on 54 (4) (2007) 811 -816.

[3] S. Awadalla, J. Mackenzie, H. Chen, B. Redden, G. Bindley, M. Duff, A. Burger, M. Groza, V. Buliga, J. Bradley, Z. Dai, N. Teslich, D. Black, Characterization of detector-grade CdZnTe crystals grown by traveling heater method (THM), Journal of Crystal Growth 312 (4) (2010) 507 - 513. 
[4] P. Rudolph, M. Mhlberg, Basic problems of vertical bridgman growth of CdTe, Materials Science and Engineering: B 16 (13) (1993) 8 - 16.

[5] P. Rudolph, Fundamental studies on bridgman growth of CdTe, Progress in Crystal Growth and Characterization of Materials 29 (1994) 275 - 381.

[6] J. Greenberg, P-T-X phase equilibrium and vapor pressure scanning of nonstoichiometry in CdTe, Journal of Crystal Growth 161 (1996) 1 - 11.

[7] A. E. Mokri, R. Triboulet, A. Lusson, A. Tromson-Carli, G. Didier, Growth of large, high purity, low cost, uniform CdZnTe crystals by the cold travelling heater method, Journal of Crystal Growth $168-174$.

[8] Y. Wang, K. Kudo, Y. Inatomi, R. Ji, T. Motegi, Growth interface of CdZnTe grown from Te solution with THM technique under static magnetic field, Journal of Crystal Growth 284 (2005) $406-411$.

[9] U. Roy, S. Weiler, J. Stein, Growth and interface study of 2 in diameter CdZnTe by THM technique, Journal of Crystal Growth 312 (19) (2010) 2840 - 2845.

[10] W. W. Mullins, R. F. Sekerka, Stability of a planar interface during solidification of a dilute binary alloy, Journal of Applied Physics 35 (2) (1964) 444-451.

[11] J. H. Peterson, A. Yeckel, J. J. Derby, A comprehensive model for the growth of cadmium telluride (CdTe) by the traveling heater method (THM), Journal of Crystal Growth, to be submitted.

[12] C. W. Lan, D. T. Yang, A computer simulation of crystal growth by the travelingsolvent method (TSM): Pseudo-steady-state calculations, Modelling and Simulation in Materials Science and Engineering 3 (1) (1995) 71.

[13] C. Stelian, T. Duffar, Numerical modeling of CdTe crystallization from Te solution under terrestrial and microgravity conditions, Journal of Crystal Growth 400 (2014) 67 -75 . 
[14] Crystal Growth Laboratory, Fraunhofer IISB, CrysMAS User Manual and Tutorial, http://www.cgl-erlangen.com/downloads/Manual.pdf (2003).

[15] A. Yeckel, R. Goodwin III, Cats2D (Crystalization and Transport Simulator) User Manual, http://www.msi.umn.edu/ yeckel/cats2d.html (2003).

[16] A. Yeckel, A. Salinger, J. Derby, Theoretical analysis and design considerations for floatzone refinement of electronic grade silicon sheets, Journal of Crystal Growth 152 (1995) $51-64$.

[17] R. Goodwin, W. Schowalter, Arbitrarily oriented capillary-viscous planar jets in the presence of gravity, Physics of Fluids 7 (5) (1995) 954-963.

[18] A. Yeckel, J. Smith, J. Derby, Parallel finite element calculation of flow in a threedimensional lid-driven cavity using the CM-5 and T3D, International Journal for Numerical Methods in Fluids 24 (12) (1997) 1449-1461.

[19] N. Zhang, A. Yeckel, A. Burger, Y. Cui, K. Lynn, J. Derby, Anomalous segregation during electrodynamic gradient freeze growth of cadmium zinc telluride, Journal of Crystal Growth 325 (1) (2011) 10 - 19.

[20] G. Samanta, A. Yeckel, E. D. Bourret-Courchesne, J. J. Derby, Parametric sensitivity and temporal dynamics of sapphire crystal growth via the micro-pulling-down method, Journal of Crystal Growth 359 (2012) 99 - 106.

[21] A. Yeckel, P. Daoutidis, J. J. Derby, Stabilizing detached bridgman melt crystal growth: Model-based nonlinear feedback control, Journal of Crystal Growth 361 (2012) 16 - 24.

[22] P. Daggolu, A. Yeckel, C. E. Bleil, J. J. Derby, Stability limits for the horizontal ribbon growth of silicon crystals, Journal of Crystal Growth 363 (2013) $132-140$.

[23] T. H. Bell, Lee waves in stratified flows with simple harmonic time dependence, Journal of Fluid Mechanics 67 (1975) 705-722. 
[24] C. J. Nappo, Fundamentals, in: C. J. Nappo (Ed.), An Introduction to Atmospheric Gravity Waves, Vol. 102 of International Geophysics, Academic Press, 2012, pp. 29 56.

[25] J. H. Peterson, M. Divecha, J. J. Derby, Lee wave formation during the growth of cadmium telluride (CdTe) by the traveling heater method (THM), Physics of Fluids, to be submitted. 


\section{Global Model}

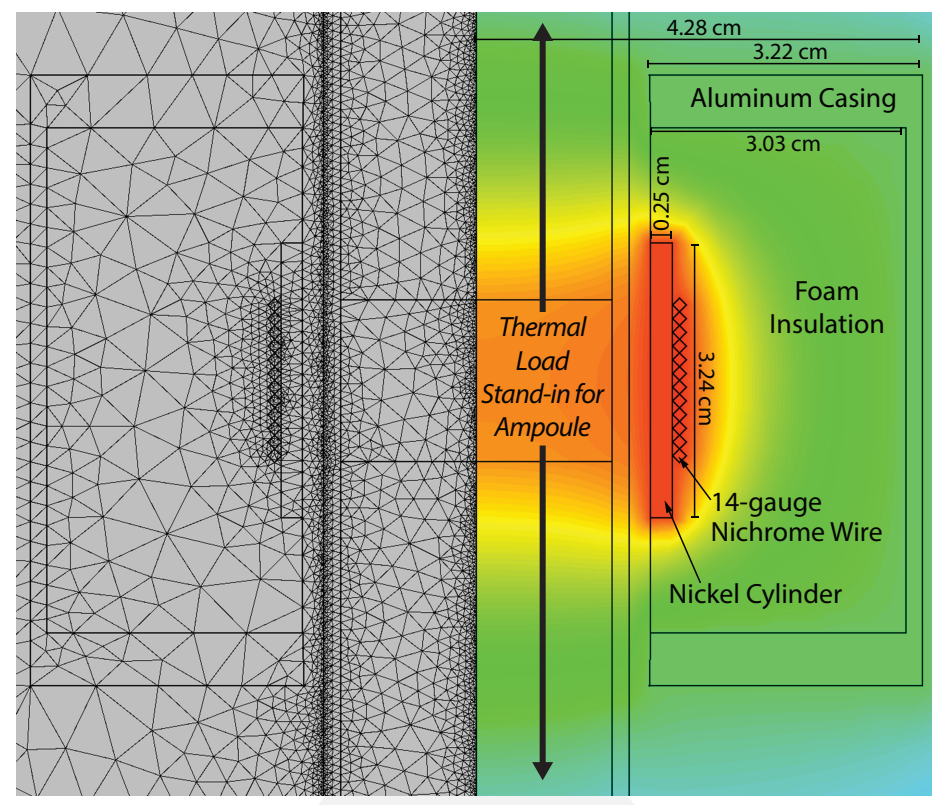

$\checkmark$

\section{Coupling through radiation heat} transfer boundary condition

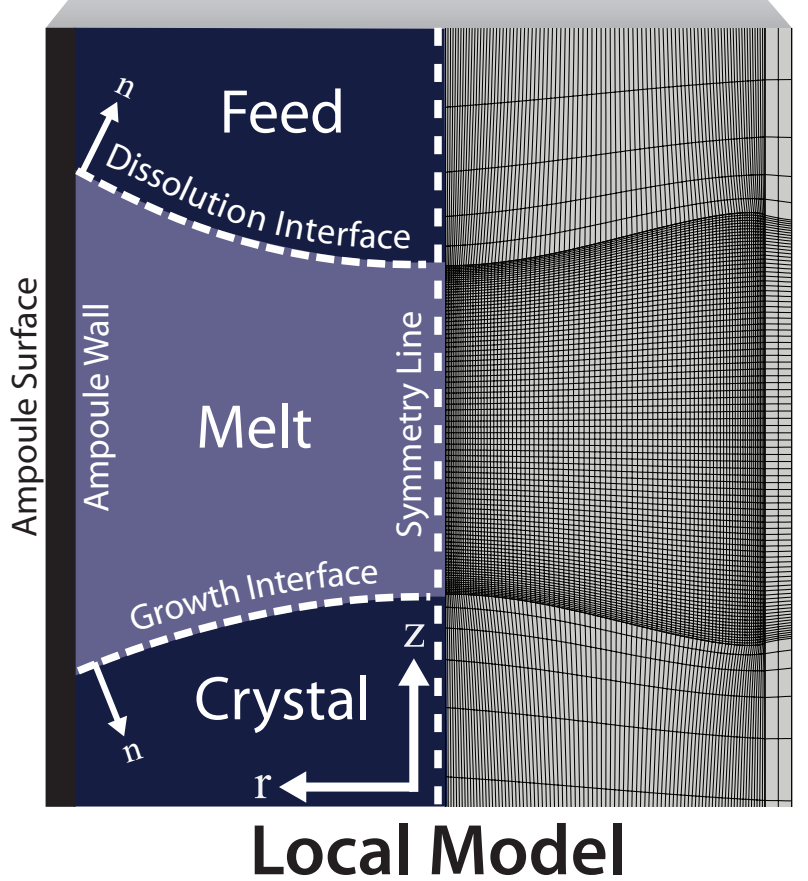

Figure 1: Top: A global model for heat transfer in the furnace is used to provide thermal boundary conditions to the local model. Bottom: Schematic and mesh representation of the local model of ampoule and contents. 


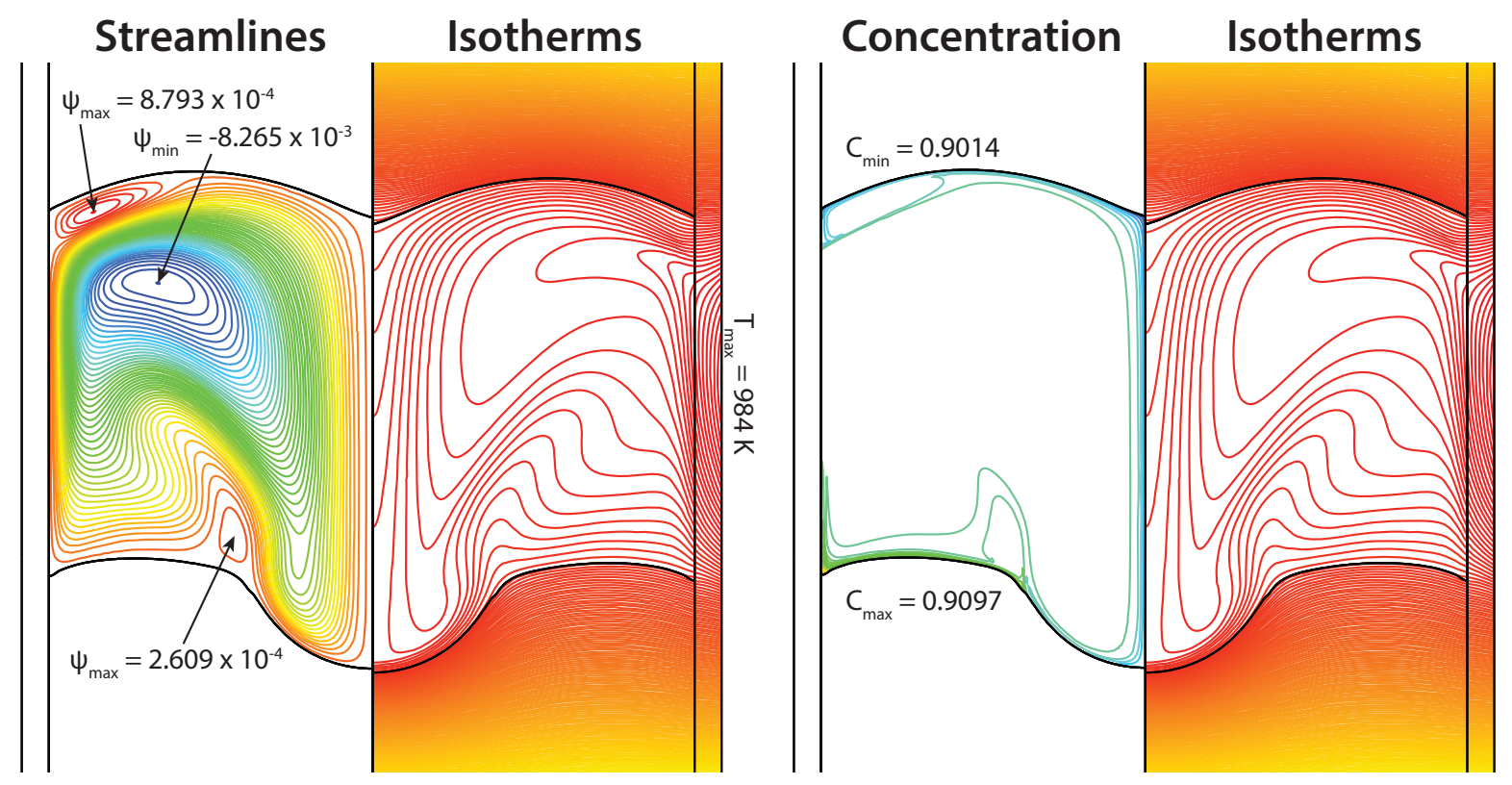

Figure 2: Streamline, isotherm, and iso-concentration contour plots for THM growth of CZT at a growth rate of $1.181 \mathrm{~mm} /$ day and a vertical gradient at the growth interface of approximately $75 \mathrm{~K} / \mathrm{cm}$. Isotherms are spaced at intervals of $1.18 \mathrm{~K}$, and concentration is displayed in mole fraction of the tellurium solvent.

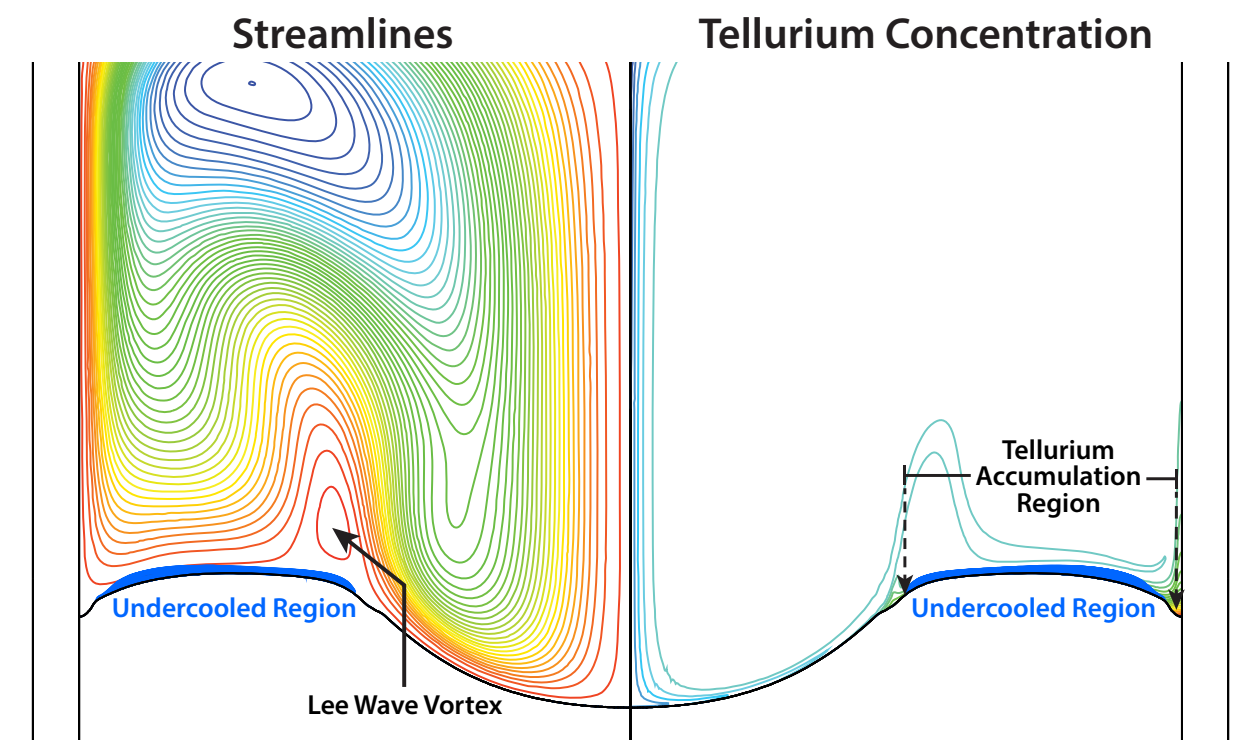

Figure 3: Streamlines and tellurium iso-concentration lines for THM growth of CZT at a growth rate of 1.475 $\mathrm{mm} /$ day and a vertical gradient at the growth interface of approximately $55 \mathrm{~K} / \mathrm{cm}$. The blue overlay shows regions in the liquid where undercooling exists, computed as a function of local temperature and composition from the phase diagram. Supercooling occurs behind the lee-wave vortex. 

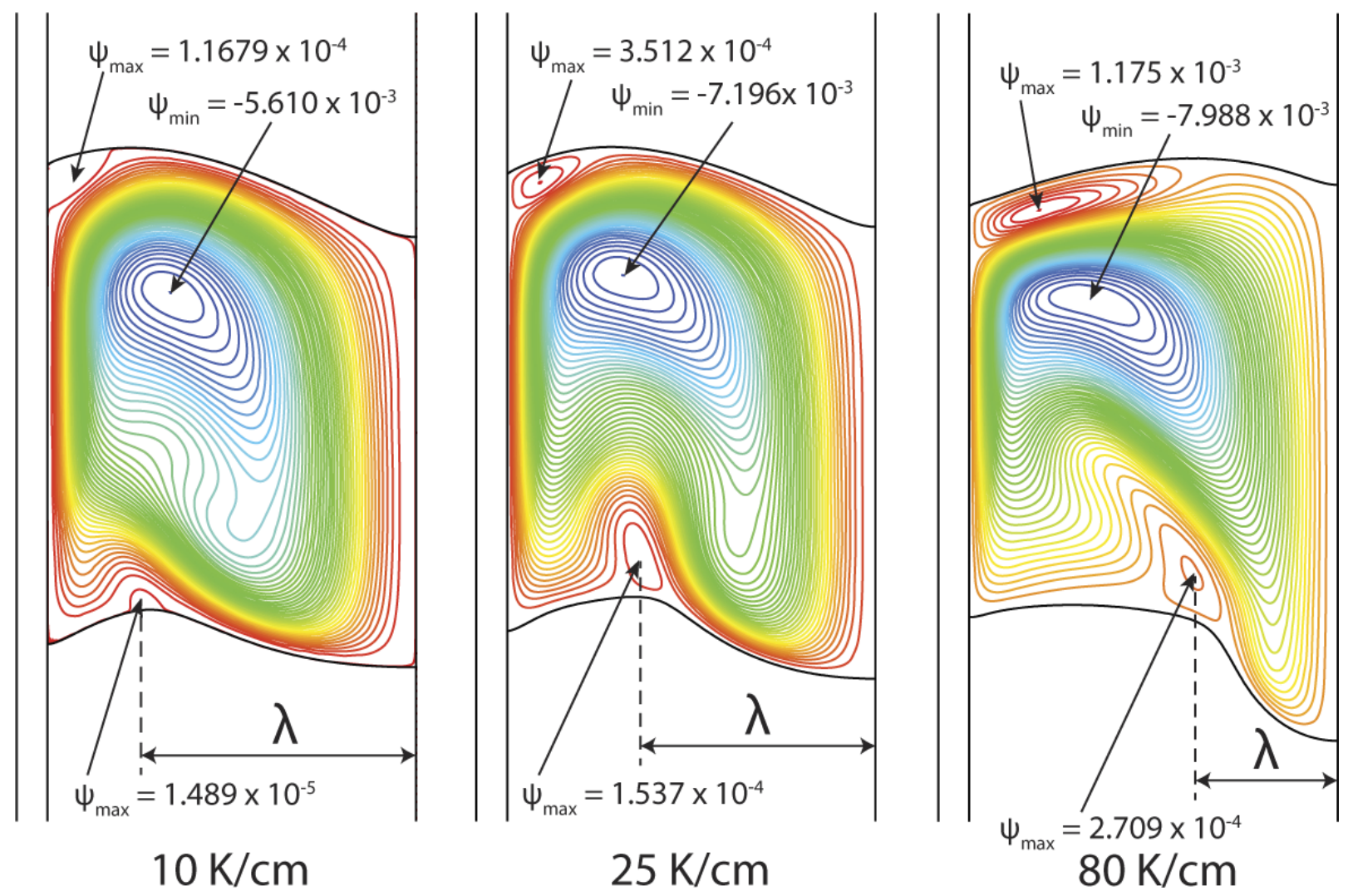

Figure 4: Streamlines are plotted as the vertical thermal gradient near the growth interface is varied. The radial position of the counter-rotating, lee-wave vortex at the growth interface is depicted by the wavelength $\lambda$ and is shown to decrease as the thermal gradient increases. Isotherms are spaced at intervals of $1.18 \mathrm{~K}$ while the spacing between streamlines is varied to show 50 stream contours. The local minima and maxima of the streamfunction $\psi$ are also shown; these values scale with total volumetric flow, indicating the relative strength of the flow vortices. 